\title{
Neutrophil-to-Lymphocyte Ratio and
}

\section{Platelet-to-Lymphocyte Ratio Predict Mortality in Patients with Diabetic Foot Ulcers Undergoing Amputations}

\author{
Wenwen Chen ${ }^{1, *}$ \\ Kun Chen $\left(\mathbb{D}^{2, *}\right.$ \\ Zhixiao $\mathrm{Xu}^{3}$ \\ Yepeng $\mathrm{Hu}^{\prime}$ \\ Yiying Liu' \\ Wenyue Liu' \\ Xiang $\mathrm{Hu}^{\prime}$ \\ Tingting $\mathrm{Ye}^{\mathrm{I}}$ \\ Jing Hong (D) \\ Hong Zhu' \\ Feixia Shen' \\ 'Department of Endocrinology and \\ Metabolism, The First Affiliated Hospital \\ of Wenzhou Medical University, \\ Wenzhou, People's Republic of China; \\ ${ }^{2}$ Department of Thoracic Surgery, The \\ First Affiliated Hospital of Wenzhou \\ Medical University, Wenzhou, People's \\ Republic of China; ${ }^{3}$ Department of \\ Pulmonary and Critical Care Medicine, \\ The First Affiliated Hospital of Wenzhou \\ Medical University, Wenzhou, People's \\ Republic of China
}

*These authors contributed equally to this work

Correspondence: Feixia Shen Department of Endocrinology and Metabolism, The First Affiliated Hospital of Wenzhou Medical University, Wenzhou, People's Republic of China Email shenfeixia@wmu.edu.cn
This article was published in the following Dove Press journal: Diabetes, Metabolic Syndrome and Obesity: Targets and Therapy

Purpose: Elevated platelet-to-lymphocyte ratio (PLR) and neutrophil-to-lymphocyte ratio (NLR) are associated with poor outcomes in various diseases. The objectives of this study were to explore the utility of PLR and NLR in predicting all-cause mortality in patients with diabetic foot ulcers (DFU) undergoing amputations.

Patients and Methods: A retrospective observational study was performed that included a total of 348 DFU patients undergoing amputations. The primary end-point was all-cause death. According to the PLR and NLR cut-off values, patients were divided into two groups and Kaplan-Meier survival curves were constructed. Multivariable Cox regression was conducted to test the independent predictors of mortality in the study cohort.

Results: All-cause mortality was significantly higher in patients with a high PLR/NLR compared to those with a low PLR/NLR. In the low NLR group, the overall survival (OS) rates at 1,3 , and 5 years after amputation were $96.8 \%, 84 \%$ and $80.1 \%$, respectively ( $p=0.001$ ). In the high NLR group the corresponding OS rates at 1,3 , and 5 years were $85.2 \%, 58.6 \%$ and $23.9 \%(p<0.001)$. According to the multivariate analysis, age (HR 1.074 95\% CI 1.045-1.104, p<0.001), Wagner classification (HR 2.274, 95\% CI 1.351-3.828, $\mathrm{p}=0.002)$, PLR (HR 1.794, 95\% CI 1.014-3.174, $\mathrm{p}=0.045)$, NLR (HR 2.029, 95\% CI 1.177-3.499, $\mathrm{p}=0.011$ ), creatinine (HR 1.003, 95\% CI 1.001-1.004, $\mathrm{p}<0.001)$ and direct bilirubin (HR 1.154, 95\% CI 1.081-1.232, p<0.001) were independent predictors of mortality following amputation.

Conclusion: Postoperative PLR and NLR values may be reliable predictive biomarkers of mortality in patients following amputation for DFU. Considering the high mortality in those patients, the patients with elevated PLR/NLR should be given more intensive in clinical practice.

Keywords: amputation, platelet-to-lymphocyte ratio, PLR, neutrophil-to-lymphocyte ratio, NLR, diabetic foot ulcer, mortality

\section{Introduction}

The prevalence of diabetes has been rising rapidly throughout the world. In 2019, the International Diabetes Federation (IDF) estimated that the prevalence of diabetes in adults aged 18-99 years was approximately 9.3\% and further predicted this to rise to $10.9 \%$ by $2045 .{ }^{1}$ Foot ulcer is a major complication of diabetes and the risk of developing a diabetic foot ulcer (DFU) is around $25 \%$ during the lifetime of a diabetic patient. ${ }^{2}$ 
Moreover, DFUs are associated with higher rates of lower extremity amputation (LEA) with the rate being 15-to 40fold higher than in patients with DFUs compared to the general population. ${ }^{3}$ The mortality rate of patients following amputation is also very high and at 5 years can reach $80 \%$ which is worse than that observed in several types of cancers. $^{4,5}$ Improving the management of patients with DFUs remains critical towards reducing the mortality rate of patients and improved biomarkers to predict mortality rates following amputation due to DFUs are urgently required.

Neutrophil-lymphocyte ratio (NLR) and platelet-tolymphocyte ratio (PLR) are novel biomarkers of systemic inflammation that can be obtained from routine blood examinations and can be easily implemented into clinical practice. It has been demonstrated that PLR and NLR are significant inflammatory markers that can predict mortality in populations suffering from cardiovascular diseases and cancers. ${ }^{6-9}$ The exact mechanism through which high PLR/NLR results in enhanced mortality remains unclear yet it is most likely that inflammation plays an important role. Recently, PLR and NLR have also been reported to have predictive power in diabetic complications. ${ }^{10,11}$ To our best of knowledge, the association between PLR/NLR and all-cause mortality after amputation in DFU patients has not yet been reported. This study aimed to determine the potential role of PLR and NLR as predictors of mortality in DFU patients following LEA.

\section{Patients and Methods Study Population}

This retrospective cohort study included 348 adult patients to explore the predictive value of NLR and PLR on allcause mortality in DFU patients following LEA. For our analysis, the following inclusion criteria were used: (1) patients diagnosed with type 2 diabetes mellitus and diabetic foot ulcers, and (2) patients who had consented to receive amputation at The First Affiliated Hospital of Wenzhou Medical University between 2015 and 2019. Patients were excluded according to the following criteria: (1) patients who lacked laboratory or follow-up data, (2) patients with severe systemic infections or blood diseases that affect neutrophils and lymphocytes, and (3) patients with complications including serious dysfunctions of the heart, lung, kidney, brain and other organs.

\section{Methods and Calculations}

Three hundred and forty-eight patients were eligible for inclusion in the study. The primary end-point was all- cause death and these data were obtained from medical records or by telephone interviews. Patient data including demographic variables (age and sex), anthropometric parameters (height and weight), type of diabetes, disease duration, history of previous amputations, history of smoking, and alcohol abuse were collected from individual medical records upon admission according to specified definitions. All patients were examined to grade the severity of infection according to the Infectious Diseases Society of America and the International Working Group on the Diabetic Foot (IDSA-IWGDF) criteria. Foot ulcers were graded according to the Wagner's classification ${ }^{12}$ which was used according to the ulcer depth and the presence of osteomyelitis or gangrene. Amputations below the ankle were classified as minor amputations whilst higher amputations were defined as major amputations. PLR was calculated as the ratio of platelets to lymphocytes and NLR calculated as the ratio of the neutrophil to lymphocytes. BMI was calculated as body weight divided by the square of the height.

\section{Statistical Analysis}

Continuous variables are presented as the mean \pm SD and were compared using a Student's $t$-test. The categorical variables are presented as frequencies and percentages and were analyzed using the Pearson's $\chi 2$-test. The predictive values of PLR and NLR for the primary endpoint were also evaluated by calculating the area under the curve (AUC) from the receiver operating characteristic (ROC) curves. Patients were divided into two groups based on the cut-off values of the PLR and NLR. Survival analysis was estimated using the KaplanMeier survival curves and differences between the survival curves assessed using a Log rank test. Univariate and multivariate survival analyses were conducted using the Cox proportional hazards model. In the multivariate Cox model, the predictor was included based on the score and the best selection criteria. The independent association between PLR/NLR and mortality were identified by multivariate Cox regression analysis and the variables which showed significant associations with survival in univariate Cox analysis were included. All statistical analyses were performed using the IBM SPSS 25.0 software for Windows. Statistical tests and 95\% confidence intervals (CIs) were 2-sided, with a significance level of 0.05 . 


\section{Results}

\section{Baseline Demographic and Clinical Characteristics}

Three hundred and forty-eight patients were recruited to the study that included 228 males and 120 females, with mean ages of $65.37 \pm 9.61$ years and $68.23 \pm 9.51$ years, respectively. Amongst all patients, 314 (90.23\%) had peripheral arterial disease, 269 (77.29\%) patients had hypertension, $25(7.2 \%)$ patients had coronary heart disease, 34 $(9.7 \%)$ patients had cerebral vascular disease, $119(34 \%)$

Table I Summary of Baseline Patient Characteristics

\begin{tabular}{|c|c|c|c|c|c|c|}
\hline & High PLR N=200 & Low PLR N= I 48 & & High NLR N= I 55 & Low NLR N=193 & \\
\hline Clinical Parameters & $\mathbf{N}(\mathbf{N} \%$ or SD) & $\mathbf{N}(\mathbf{N} \%$ or SD) & p-value & $\mathbf{N}(\mathbf{N} \%$ or SD) & $\mathbf{N}(\mathbf{N} \%$ or SD) & p-value \\
\hline Age & $67.31 \pm 9.49$ & $65.07 \pm 9.77$ & 0.033 & $68.11 \pm 9.74$ & $64.95 \pm 0.68$ & 0.002 \\
\hline Sex & & & 0.813 & & & 0.216 \\
\hline Male & $130(65 \%)$ & $98(66.21 \%)$ & & $107(69.03 \%)$ & $|2|(62.69 \%)$ & \\
\hline Female & $70(35 \%)$ & $50(33.78 \%)$ & & $48(30.97 \%)$ & $72(37.3 \%)$ & \\
\hline BMI, $\mathrm{kg} / \mathrm{m}^{2}$ & $22.65 \pm 6.25$ & $23.64 \pm 4.02$ & 0.101 & $23.04 \pm 5.24$ & $23.04 \pm 5.51$ & 0.852 \\
\hline SBP $(\mathrm{mmHg})$ & $|45.3| \pm 22.86$ & $142.46 \pm 24.40$ & 0.269 & $143.87 \pm 24.03$ & $144.28 \pm 23.18$ & 0.873 \\
\hline $\mathrm{DBP}(\mathrm{mmHg})$ & $74.98 \pm|2.9|$ & $76.18 \pm 12.55$ & 0.383 & $74.84 \pm 13.66$ & $76.01 \pm 13.66$ & 0.398 \\
\hline Duration of diabetes (years) & $12.46 \pm 8.22$ & $12.16 \pm 7.2$ & 0.417 & $13.18 \pm 8.286$ & $11.65 \pm 7.296$ & 0.007 \\
\hline Coronary heart disease & $16(8 \%)$ & $9(6.08 \%)$ & 0.493 & $12(7.74 \%)$ & $13(6.73 \%)$ & 0.436 \\
\hline Cerebral vascular disease & $20(10 \%)$ & $14(9.45 \%)$ & 0.867 & $19(12.25 \%)$ & $15(7.77 \%)$ & 0.158 \\
\hline Lower extremity arterial diseases & $180(90 \%)$ & $130(90.5 \%)$ & 0.867 & 138(89.03\%) & $176(91.19 \%)$ & 0.500 \\
\hline Wagner classification & & & 0.01 & & & 0.110 \\
\hline 2 and 3 & $79(39.5 \%)$ & $79(53.37 \%)$ & & $63(40.65 \%)$ & $95(49.22 \%)$ & \\
\hline 4 and 5 & $|2|(60.5 \%)$ & $69(46.63 \%)$ & & $92(59.35 \%)$ & $98(50.78 \%)$ & \\
\hline Smoking history (current or ever) & $67(33.5 \%)$ & $52(35.1 \%)$ & 0.751 & $54(34.83 \%)$ & $65(33.68 \%)$ & 0.821 \\
\hline Prior history of amputation & $23(14.7 \%)$ & $10(8.3 \%)$ & 0.168 & $16(10.32 \%)$ & $22(11.39 \%)$ & 0.749 \\
\hline \multicolumn{7}{|l|}{ Laboratory data } \\
\hline $\mathrm{HbAIc} \%$ & $9.49 \pm 2.29$ & $9.63 \pm 2.39$ & 0.598 & $9.42 \pm 2.38$ & $9.62 \pm 2.25$ & 0.472 \\
\hline FBG & $8.99 \pm 3.11$ & $8.57 \pm 3.15$ & 0.265 & $9.30 \pm 3.26$ & $8.56 \pm 3.10$ & 0.033 \\
\hline White blood cell & $7.06 \pm 2.66$ & $6.57 \pm 1.98$ & 0.055 & $8.22 \pm 2.60$ & $5.826 \pm 1.60$ & 0.004 \\
\hline Mean platelet volume & $9.83 \pm 1.02$ & $10.53 \pm 1.09$ & $<0.001$ & $9.94 \pm 1.15$ & $10.29 \pm 1.06$ & $<0.001$ \\
\hline Fibrinogen & $7.16 \pm 1.82$ & $6.09 \pm 1.90$ & $<0.001$ & $6.95 \pm 1.84$ & $6.51 \pm 1.97$ & 0.037 \\
\hline Total bilirubin & $7.48 \pm 3.15$ & $8.39 \pm 5.21$ & 0.062 & $7.46 \pm 3.6$ & $8.19 \pm 4.57$ & 0.103 \\
\hline Direct bilirubin & $3.45 \pm 1.94$ & $3.8 I \pm 3.28$ & 0.195 & $3.55 \pm 2.79$ & $3.64 \pm 2.43$ & 0.738 \\
\hline Indirect bilirubin & $4.03 \pm 1.74$ & $4.57 \pm 2.79$ & 0.38 & $3.91 \pm 1.60$ & $4.54 \pm 2.65$ & 0.006 \\
\hline Albumin & $30.7 \pm 5.39$ & $32.24 \pm 4.7 I$ & 0.006 & $30.3 \pm 4.97$ & $32.19 \pm 5.17$ & 0.001 \\
\hline Creatinine & $|36.5| \pm 163.12$ & $108.01 \pm 141.7$ & 0.083 & $158.65 \pm 189.36$ & $96.87 \pm 113.33$ & $<0.001$ \\
\hline Uric acid & $295.03 \pm 118.54$ & $294.11 \pm 99.2$ & 0.939 & $314.65 \pm 124.56$ & $278.50 \pm 95.23$ & 0.003 \\
\hline Blood urea nitrogen & $7.73 \pm 7.94$ & $6.674 \pm 3.71$ & 0.024 & $8.4 I \pm 5.25$ & $6.37 \pm 3.51$ & $<0.001$ \\
\hline
\end{tabular}

Note: Data are presented as the means \pm standard deviation (SD), the median (interquartile range) for continuous variables or the number (\%) for categorical variables. Abbreviations: BMI, body mass index; SBP, systolic blood pressure; DBP, diastolic blood pressure; FBG, fasting blood glucose. 
patients were smokers, 102 (29.1\%) patients were drinkers and $38(10.9 \%)$ patients had prior histories of amputation.

The mean values of PLR and NLR were $189.96 \pm 87.10$, and $3.21 \pm 2.59$, respectively. According to the cut-off value of the PLR, 148 (42.53\%) subjects were included in the low PLR group (PLR $<160.05)$, whereas the remaining 200 (57.47\%) subjects were included the high PLR group (PLR $\geq 160.05$ ). From the cut-off value of the NLR, two groups were defined as the high (NLR $\geq 2.76)$ and low $(\mathrm{NLR}<2.76)$ NLR groups that contained 155 and 193 patients, respectively. The demographic characteristics and laboratory findings of patients are summarized in Table 1.

The results indicated that when the patients were separated into two groups according to the PLR cut-off value, significant differences were observed in the Wagner classification $(p=0.01)$, mean platelet volume (MPV) $(p<0.001)$, fibrinogen $(p<0.001)$, albumin $(p=0.006)$. Fibrinogen and the grade of Wagner classification were significantly higher in the high PLR group, whilst albumin and MPV were significantly higher in the low PLR group. All other parameters were not statistically different across the patient groups.

When the patients were separated into two groups according to the NLR cut-off value, significant differences were observed in the following variables: age, duration of diabetes mellitus, fasting blood glucose (FBG), white blood cell (WBC), mean platelet volume (MPV),

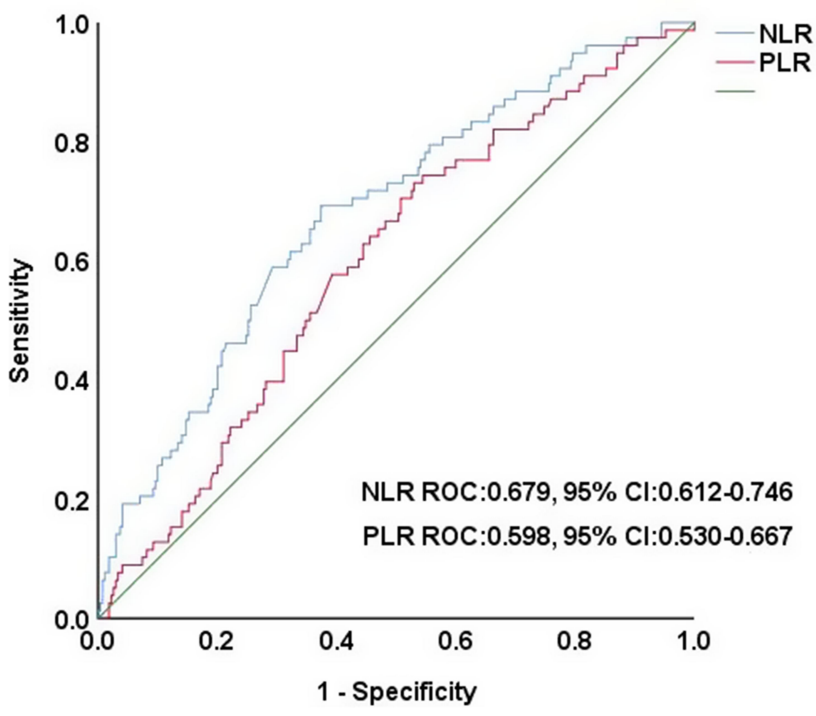

Figure I ROC curves for predicting the primary endpoint (all-cause mortality) for baseline NLR and PLR. The optimum cut-off values were NLR $\geq 2.76$ (sensibility 69.2\%, specificity $62.6 \%$ ) and PLR $\geq 160.05$ (sensibility $73.1 \%$, specificity $47 \%$ ). Abbreviations: ROC, receiver operating characteristic; NLR, neutrophil-tolymphocyte ratio; PLR, platelet-to-lymphocyte ratio. fibrinogen, albumin, creatinine, blood urea nitrogen (BUN), and uric acid (UA). In the high NLR group, age, FBG, and WBC were significantly higher and the duration of diabetes was longer compared to the low NLR group. Also, WBC, fibrinogen, creatinine, and uric acid were significantly higher in the high PLR group. Albumin, indirect bilirubin and MPV were significantly higher in the low NLR group. All other parameters were not statistically different across the patient groups.

ROC curve analysis suggested that the optimum PLR (AUC $=0.598,95 \%$ CI $0.530-0.667)$ cut-off point for predicting mortality was 160.05 , with a sensitivity of $73.1 \%$ and specificity of $47 \%$. Similar analysis showed the optimum NLR (AUC $=0.679,95 \%$ CI $0.612-0.746$ ) cut-off point for predicting mortality was 2.76, with a sensitivity of $69.2 \%$ and specificity of $62.6 \%$ (Figure 1 ).

\section{Predictors of Mortality After Amputation}

A Log rank test of the Kaplan-Meier curves indicated that patients in the high PLR and NLR groups had a lower OS rate compared to patients in the low PLR and NLR groups (Figure 2). In the low NLR group, OS rates at 1, 3, and 5 years after amputation were $96.8 \%, 84 \%, 80.1 \%$, respectively. In the high NLR group, the corresponding OS rates at 1,3 , and 5 years after amputation were $85.2 \%, 58.6 \%$ and $23.9 \%(p<0.001)$. The median survival time in the high NLR group was $50 \pm 3.68$ months $(95 \%$ CI $42.78-53.22)$. The OS rates at 1,3 , and 5 years after amputation in low PLR group were $95.7 \%, 83.9 \%$ and $74.8 \%$, respectively. In the high PLR group, the corresponding OS rates at 1, 3, and 5 years after amputation were $88.6 \%, 64.5 \%$ and $47.6 \%$ $(p=0.001)$. Patients were also divided into two groups based on the cut-off values of the neutrophil, the reciprocal of the lymphocyte and platelet counts, respectively. Patients in the high neutrophil groups had a lower OS rate compared to patients in the low neutrophil groups. In the low reciprocal of the lymphocyte group, OS is higher, while the result of platelet was not significant (Supplementary Figure 1).

As shown in Table 2, univariate Cox regression analysis suggested that OS was associated with the following variables: age, Wagner classification, creatinine, systolic blood pressure (SBP), PLR, NLR, direct bilirubin, UA, blood urea nitrogen (BUN). After adjusting other covariates, the HR of UA, BUN and SBP became nonsignificant, whereas the direct bilirubin became significant. The age, Wagner classification, PLR, NLR, creatinine remained significant predictors in the multivariate models. 
A

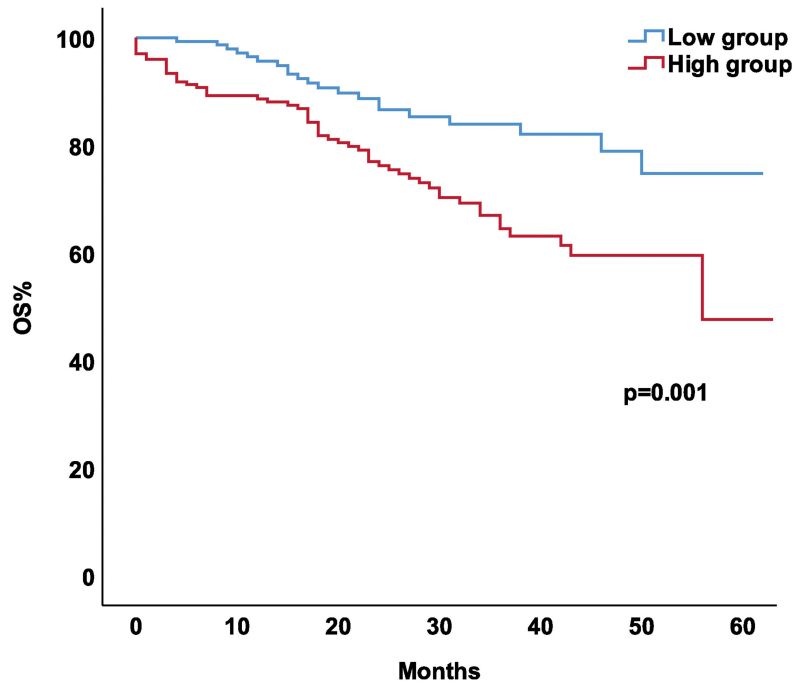

B

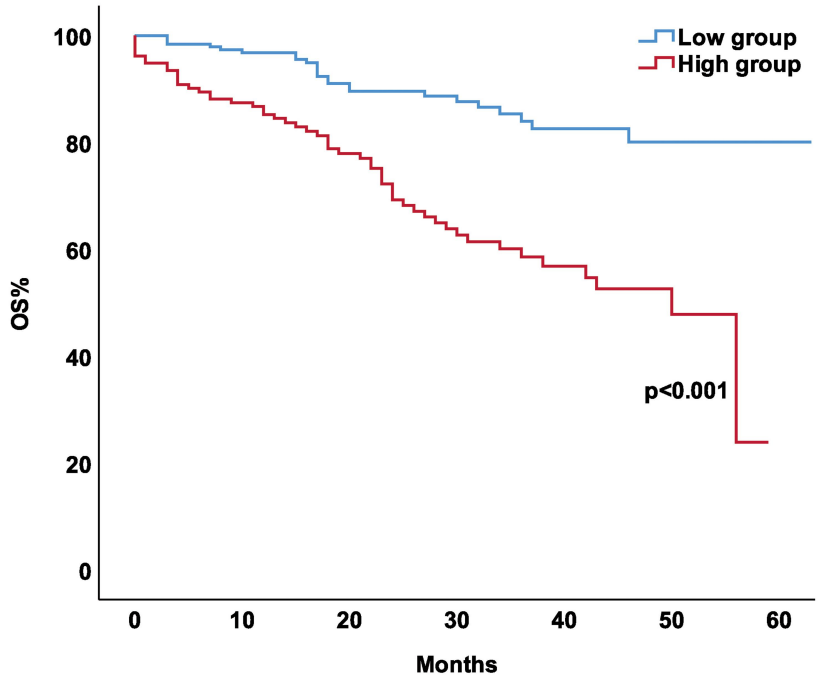

Figure 2 Kaplan-Meier analysis showing cumulative mortality according to the optimal cut off value. (A) Platelet-to-lymphocyte ratio (I60.05). (B) Neutrophil-tolymphocyte ratio (2.76).

Abbreviation: OS, overall survival.

\section{Discussion}

This retrospective study investigated the values of postoperative PLR and NLR in predicting all-cause death following amputation in DFU. As expected, the patients undergoing LEA in our study had high mortality (mean survival 48.946 \pm 1.369 months), which is consistent with previously reported $^{13-15}$. Thus, the independent prognostic factors that used for predicting mortality in DFU patients undergoing LEA are vital. Considering the association of postoperative NLR and PLR with higher mortality, we purpose using postoperative PLR and NLR as independent prognostic markers to predict mortality in DFU patients LEA.

In agreement with previous studies, we demonstrated that increased age, higher Wagner grade and renal disease are associated with mortality in diabetic patients after LEA. ${ }^{16-18}$ In the multivariate Cox regression analysis, we have adjusted the age, Wagner classification, creatinine, PLR and NLR. Although patients in the higher NLR/PLR group were older with a higher burden of comorbidities, this association remained significant in multivariate analysis. We also found that direct bilirubin was independently associated with mortality (HR 1.154, 95\% CI 1.081-1.232, $\mathrm{p}=0.006$ ).

As a potent endogenous antioxidant, bilirubin inhibits lipid peroxidation and is associated with diabetes and many diabetic complications in several cross-sectional studies. ${ }^{19,20}$ However, the potential protective effect of bilirubin in diabetes and diabetic complications remains controversial.
Many studies have focused on total bilirubin function and have not differentiated direct and indirect bilirubin from total bilirubin. Chen et al demonstrated that indirect but not direct bilirubin were independent risk factors for the severity of diabetic foot ${ }^{20}$ whilst Wang et al found that direct bilirubin but not indirect bilirubin levels were associated with increased risk of type $2 \mathrm{DM}^{21}$ Accordingly, further studies are required to accurately determine the differential effects of different types of bilirubin.

PLR and NLR are increasingly recognized as systemic markers of overall inflammation. ${ }^{22}$ Inflammation, procoagulant imbalance and endothelial dysfunction play important roles in the development of diabetes and diabetic complications. Inflammatory disorders often cause tissue damage, microangiopathy and macrovascular complications in diabetic patients, ${ }^{10,23,24}$ often leading to end-organ damage that is associated with mortality. Also, cardiovascular events are a frequent cause for mortality in patients with DM. ${ }^{25} \mathrm{DFU}$ is a complex generalized disease that is the main reason for LEA in diabetic patients and patients requiring LEA often suffer from more severe cardiovascular diseases. ${ }^{26}$ There is also a close connection between mortality and infectious complications such as, sepsis, pneumonia. DFU patients with LEA, always come with tissue necrosis, systemic infection, and inflammation, which can increase mortality. Based on these observations, the adoption of PLR and NLR should be considered as important predictive tools of mortality in these patients. 
Table 2 Univariate and Multivariate Analyses of the Overall Survival of Patients with Diabetic Foot Ulcers Undergoing Amputations

\begin{tabular}{|c|c|c|c|c|}
\hline \multirow[t]{2}{*}{ Covariate } & \multicolumn{2}{|l|}{ Univariate } & \multicolumn{2}{|l|}{ Multivariate } \\
\hline & HR (95\% Cl) & p-value & HR (95\% Cl) & p-value \\
\hline Age & $1.072(1.045-1.099)$ & $<0.001$ & $1.074(1.045-1.104)$ & $<0.001$ \\
\hline \multicolumn{5}{|l|}{ Sex } \\
\hline Male & 1 & & - & - \\
\hline Female & $0.960(0.597-1.545)$ & 0.868 & - & - \\
\hline Coronary heart disease & $1.360(0.653-2.830)$ & $0.4 I I$ & - & \\
\hline Cerebral vascular disease & $0.862(0.375-1.985)$ & 0.728 & - & - \\
\hline \multicolumn{5}{|l|}{ Wagner classification } \\
\hline 2 and 3 & 1 & & - & - \\
\hline 4 and 5 & $2.366(I .443-3.878)$ & $<0.001$ & $2.274(1.35 I-3.828)$ & 0.002 \\
\hline Smoking history (current or ever) & $0.700(0.427-1.147)$ & 0.157 & - & - \\
\hline Prior history of amputation & $1.002(0.515-1.948)$ & 0.996 & - & - \\
\hline Systolic blood pressure $(\mathrm{mmHg})$ & $1.017(1.005-1.028)$ & 0.005 & $1.007(0.997-1.018)$ & 0.149 \\
\hline Diastolic blood pressure $(\mathrm{mmHg})$ & $1.003(0.984-1.022)$ & 0.775 & - & - \\
\hline$P L R \geq|55.4|$ & $3.335(2.059-5.402)$ & $<0.001$ & $1.794(1.014-3.174)$ & 0.045 \\
\hline$N L R \geq 3.06$ & $2.224(1.347-3.670)$ & 0.002 & $2.029(1.177-3.499)$ & 0.011 \\
\hline FBG & $0.962(0.888-1.043)$ & 0.347 & - & - \\
\hline Platelet & $1.000(0.998-1.002)$ & 0.840 & - & - \\
\hline Mean platelet volume & $0.952(0.780-1.162)$ & 0.627 & - & - \\
\hline Fibrinogen & $1.052(0.934-1.185)$ & 0.399 & - & - \\
\hline Total bilirubin & $1.007(0.953-1.064)$ & 0.797 & - & - \\
\hline Direct bilirubin & $1.069(0.998-1.144)$ & 0.055 & I.I54 (I.08I-I.232) & $<0.001$ \\
\hline Indirect bilirubin & $0.909(0.796-1.038)$ & 0.159 & - & - \\
\hline Albumin & $0.984(0.943-1.028)$ & 0.474 & - & - \\
\hline Creatinine & $1.002(1.002-1.003)$ & $<0.001$ & $1.003(1.001-1.004)$ & $<0.001$ \\
\hline Uric acid & $1.003(1.002-1.005)$ & $<0.001$ & $1.001(0.999-1.003)$ & 0.277 \\
\hline BUN & $1.086(1.052-1.122)$ & $<0.001$ & $0.998(0.93 I-I .07 I)$ & 0.964 \\
\hline
\end{tabular}

Note: In the multivariate model, the following variables were added as independent variables: age, Wagner classification, PLR, NLR, creatinine, direct bilirubin, uric acid and blood urea nitrogen.

Abbreviations: HR, hazard ratio; Cl, confidence interval; SBP, systolic blood pressure; PLR, platelet-to-lymphocyte ratio; NLR, neutrophil-to-lymphocyte ratio; BUN, blood urea nitrogen; FBG, fasting blood glucose.

Growing evidence has shown that PLR and NLR are associated with an increase in all-cause mortality risk in the general population and patients with cardiovascular disease and cancers. ${ }^{8,9,27,28}$ In the present study, it was shown that patients with elevated postoperative PLR or NLR were independently associated with increased risk of mortality. Most studies that have evaluated NLR and PLR for mortality have demonstrated the predictive value of these markers. Zeng et al demonstrated that elevated PLR was independently associated with an increased 5-year allcause mortality risk in patients with chronic kidney disease (CKD). ${ }^{29}$ Hudzik et al reported that the PLR is an independent risk factor for early and late mortality in patients with DM..$^{30}$ The results of the current study are consistent 
with other reports demonstrating that higher NLR and PLR values are associated with increased mortality rates.

A high PLR occurs when the platelet counts become high or when lymphocyte count becomes low. In general, high platelet counts are associated with increased platelet activity. ${ }^{31}$ Studies have shown that increased platelet activity may reflect the aggravated release of inflammatory mediators and to promote the destructive inflammatory process. $^{32}$ High platelet counts represent increased thrombosis and the release of mediators which enhance atherosclerosis and inflammation. It may indicate ongoing inflammatory conditions and prothrombotic activities. Research findings suggest that platelet hyperactivity in parallel with thrombosis has a principal role in the pathophysiology of atherogenesis ${ }^{33}$ and the significant action in creating illness and death from atherosclerosis is due to platelet adhesion and aggregation at the site of endothelial damage or the site of rupture of atherogenic plaque. The second constituent of PLR is the lymphocyte-count which highly influences inflammatory states. During systemic inflammation, lymphocytes exert a modulatory effect on the inflammatory response and lymphocytopenia occurs as a result of accelerated apoptosis in lymphocytes. Lymphocytes could also induce the expression of interleukin-10 and promote tissue repairment. ${ }^{34}$ These results emphasize the value of PLR in predicting outcomes in LEA patients.

NLR is a biomarker that can be used to evaluate the inhibitory and excitatory activities of the immune system. Neutrophils could infiltrate vascular wall and secretion of superoxide radicals, cytokines, and a variety of proteolytic enzymes which can cause endothelial damage, whilst lymphocytes can modulate the effect of neutrophils and also have an anti-atherosclerotic role. A high NLR represents endothelial damage and dysfunction as a result of higher neutrophilic activity that can lead to worse outcomes. In the study from Dinc et al, it was shown that higher NLR was related to increased mortality in patients who underwent LEA. ${ }^{35}$ Spark et al also reported that elevated NLR is associated with higher mortality in patients with chronic critical limb ischemia (CLI). ${ }^{36}$ In our study, after adjusting for several risk factors including age, $\mathrm{Cr}$, Wagner classification, BUN, and UA, NLR could predict mortality rates in DFU patients. These results emphasize the value of NLR in assessing the inflammatory mechanisms in response to infection in predicting outcomes in LEA patients.
In the present study, we also performed a comparison of the NLR and PLR biomarkers of inflammation. Although both markers have shown predictive value in allcause mortality, few studies have directly compared their predictive power. We have found that although PLR was shown to be an independent risk factor for high mortality risk, NLR was more sensitive and was a more useful marker in the ROC curve. As shown in Figure 1, the discriminatory performance for predicting the primary endpoint was better for NLR (AUC $=0.679$, 95\% CI $0.612-0.746$ ) than for PLR (AUC $=0.598,95 \%$ CI $0.530-$ 0.667). Considering the association between PLR/NLR and worse outcomes in DFU patients undergoing LEA, PLR and NLR can be used as prognostic biomarkers, allowing physicians to generate a risk estimate of survival after LEA.

We acknowledge our study had several limitations. Firstly, the present trial was performed as a retrospective, single-center study design with a relatively small sample size. The results may therefore not accurately represent the general population of patients with diabetes-related amputations. Second, we did not compare PLR and NLR with other inflammatory markers (such as $\mathrm{C}$-reactive protein or myeloperoxidase) because they were not routinely obtained in our study. Lastly, patient information was obtained from medical records or by telephone interviews and in most cases, the cause of death could not be verified A larger and prospective study is required to highlight the clinical importance and to further validate PLR and NLR as predictive biomarkers in DFU patients.

\section{Conclusions}

In our study, we found that an increased PLR and NLR levels were reliable predictive biomarkers of mortality in DFU patients following LEA. Both of them can be easily obtained from simple complete blood count parameters in clinical practice. Considering the high mortality in DFU patients undergoing LEA, we purpose using postoperative PLR and NLR to predict mortality and the patients with elevated PLR/NLR should be given more intensive and longer duration therapy aiming to more aggressively control other risk factors.

\section{Ethic Statement}

This study has been reviewed by the ethics committee in clinical research of the First Affiliated Hospital of Wenzhou Medical University. Due to the retrospective nature of the study, the informed consent was exempted. 
In conducting this clinical study, we compliance with the ethical principles of the relevant laws, regulations and rules of China, WMA Helsinki Declaration and the ethical review measures for biomedical research involving humans (2016) of the Ministry of Health. The study followed a clinical protocol approved by local ethics committee. This study also protected the health and rights of patients and the patient data were confidentiality.

\section{Acknowledgment}

The authors thank the staff at the Department of Endocrinology and Metabolism, the First Affiliated Hospital of Wenzhou Medical University, and all the patients who participated in the study.

\section{Disclosure}

The authors report no conflicts of interest in this work.

\section{References}

1. Saeedi P, Petersohn I, Salpea P, et al. Global and regional diabetes prevalence estimates for 2019 and projections for 2030 and 2045: results from the international diabetes federation diabetes atlas, 9(th) edition. Diabetes Res Clin Pract. 2019;157:107843. doi:10.1016/j. diabres.2019.107843

2. Mavrogenis AF, Megaloikonomos PD, Antoniadou T, et al. Current concepts for the evaluation and management of diabetic foot ulcers. EFORT Open Rev. 2018;3(9):513-525. doi:10.1302/2058-5241.3.180010

3. Dutra LMA, Melo MC, Moura MC, et al. Prognosis of the outcome of severe diabetic foot ulcers with multidisciplinary care. $J$ Multidiscip Healthc. 2019;12:349-359. doi:10.2147/JMDH.S194969

4. Armstrong DG, Boulton AJM, Bus SA. Diabetic foot ulcers and their recurrence. $N$ Engl J Med. 2017;376(24):2367-2375. doi:10.1056/ NEJMra1615439

5. Jeffcoate WJ, Vileikyte L, Boyko EJ, et al. Current challenges and opportunities in the prevention and management of diabetic foot ulcers. Diabetes Care. 2018;41(4):645-652. doi:10.2337/dc17-1836

6. Proctor MJ, Morrison DS, Talwar D, et al. A comparison of inflammation-based prognostic scores in patients with cancer. A glasgow inflammation outcome study. Eur $J$ Cancer. 2011;47 (17):2633-2641. doi:10.1016/j.ejca.2011.03.028

7. Smith RA, Bosonnet L, Raraty M, et al. Preoperative platelet-lymphocyte ratio is an independent significant prognostic marker in resected pancreatic ductal adenocarcinoma. Am J Surg. 2009;197(4):466-472. doi:10.1016/j.amjsurg.2007.12.057

8. Sunbul M, Gerin F, Durmus E, et al. Neutrophil to lymphocyte and platelet to lymphocyte ratio in patients with dipper versus non-dipper hypertension. Clin Exp Hypertens. 2014;36(4):217-221. doi:10.3109/ 10641963.2013.804547

9. Azab B, Shah N, Akerman M, et al. Value of platelet/lymphocyte ratio as a predictor of all-cause mortality after non-ST-elevation myocardial infarction. J Thromb Thrombolysis. 2012;34(3):326-334. doi:10.1007/s11239-012-0718-6

10. Akbas EM, Demirtas L, Ozcicek A, et al. Association of epicardial adipose tissue, neutrophil-to-lymphocyte ratio and platelet-tolymphocyte ratio with diabetic nephropathy. Int J Clin Exp Med. 2014;7(7):1794-1801.
11. Turkmen K, Erdur FM, Ozcicek F, et al. Platelet-to-lymphocyte ratio better predicts inflammation than neutrophil-to-lymphocyte ratio in end-stage renal disease patients. Hemodial Int. 2013;17(3):391-396. doi:10.1111/hdi.12040

12. Wagner FW. The dysvascular foot: a system for diagnosis and treatment. Foot Ankle. 1981;2(2):64-122. doi:10.1177/ 107110078100200202

13. López-Valverde ME, Aragón-Sánchez J, López-de-Andrés A, et al. Perioperative and long-term all-cause mortality in patients with diabetes who underwent a lower extremity amputation. Diabetes Res Clin Pract. 2018;141:175-180. doi:10.1016/j.diabres.2018.05.004

14. Wrobel JS, Herman WH, Munson M, et al. Foot complications and mortality. J Am Podiatr Med Assoc. 2016;106(1):7-14. doi:10.7547/ 14-115

15. Lopez-de-Andres A, Jimenez-Garcia R, Esteban-Vasallo MD, et al. Time trends in the incidence of long-term mortality in T2DM patients who have undergone a lower extremity amputation. Results of a descriptive and retrospective cohort study. J Clin Med. 2019;8 (10):1597. doi: $10.3390 / \mathrm{jcm} 8101597$

16. Jupiter DC, Thorud JC, Buckley CJ, et al. The impact of foot ulceration and amputation on mortality in diabetic patients. I: from ulceration to death, a systematic review. Int Wound J. 2016;13(5):892-903. doi:10.1111/iwj.12404

17. Icks A, Scheer M, Morbach S, et al. Time-dependent impact of diabetes on mortality in patients after major lower extremity amputation: survival in a population-based 5-year cohort in Germany. Diabetes Care. 2011;34(6):1350-1354. doi:10.2337/dc10-2341

18. Margolis DJ, Hofstad O, Feldman HI. Association between renal failure and foot ulcer or lower-extremity amputation in patients with diabetes. Diabetes Care. 2008;31(7):1331-1336. doi:10.2337/ dc07-2244

19. Yang M, Ni C, Chang B, et al. Association between serum total bilirubin levels and the risk of type 2 diabetes mellitus. Diabetes Res Clin Pract. 2019;152:23-28. doi:10.1016/j.diabres.2019. 04.033

20. Chen J, Wang J, Zhang X, et al. Inverse relationship between serum bilirubin levels and diabetic foot in Chinese patients with Type 2 diabetes mellitus. Med Sci Monit. 2017;23:5916-5923. doi:10.12659/ MSM. 907248

21. Wang J, Li Y, Han X, et al. Serum bilirubin levels and risk of type 2 diabetes: results from two independent cohorts in middle-aged and elderly Chinese. Sci Rep. 2017;7(1):41338. doi:10.1038/srep41338

22. Yang W, Wang X, Zhang W, et al. Neutrophil-lymphocyte ratio and platelet-lymphocyte ratio are 2 new inflammatory markers associated with pulmonary involvement and disease activity in patients with dermatomyositis. Clin Chim Acta. 2017;465:11-16. doi:10.1016/j. cca.2016.12.007

23. Goldberg RB. Cytokine and cytokine-like inflammation markers, endothelial dysfunction, and imbalanced coagulation in development of diabetes and its complications. J Clin Endocrinol Metab. 2009;94 (9):3171-3182. doi:10.1210/jc.2008-2534

24. Forbes JM, Cooper ME. Mechanisms of diabetic complications. Physiol Rev. 2013;93(1):137-188. doi:10.1152/physrev.00045.2011

25. Durmus E, Kivrak T, Gerin F, et al. Neutrophil-to-lymphocyte ratio and platelet-to-lymphocyte ratio are predictors of heart failure. Arq Bras Cardiol. 2015;105(6):606-613. doi:10.5935/abc.20150126

26. Lee SJ, Jung YC, Jeon DO, et al. High serum C-reactive protein level predicts mortality in patients with stage 3 chronic kidney disease or higher and diabetic foot infections. Kidney Res Clin Pract. 2013;32 (4):171-176. doi:10.1016/j.krcp.2013.10.001

27. Mathur K, Kurbanova N, Qayyum R. Platelet-lymphocyte ratio (PLR) and all-cause mortality in general population: insights from national health and nutrition education survey. Platelets. 2019;30 (8):1036-1041. doi:10.1080/09537104.2019.1571188 
28. Yodying H, Matsuda A, Miyashita M, et al. Prognostic significance of neutrophil-to-lymphocyte ratio and platelet-to-lymphocyte ratio in oncologic outcomes of esophageal cancer: a systematic review and meta-analysis. Ann Surg Oncol. 2016;23(2):646-654. doi:10.1245/ s10434-015-4869-5

29. Zeng M, Liu Y, Liu F, et al. J-shaped association of platelet-tolymphocyte ratio with 5-year mortality among patients with chronic kidney disease in a prospective cohort study. Int Urol Nephrol. 2020;52(10):1943-1957. doi:10.1007/s11255-020-02548-1

30. Hudzik B, Szkodzinski J, Gorol J, et al. Platelet-to-lymphocyte ratio is a marker of poor prognosis in patients with diabetes mellitus and ST-elevation myocardial infarction. Biomark Med. 2015;9 (3):199-207. doi:10.2217/bmm.14.100

31. Kaito K, Otsubo H, Usui N, et al. Platelet size deviation width, platelet large cell ratio, and mean platelet volume have sufficient sensitivity and specificity in the diagnosis of immune thrombocytopenia. $\mathrm{Br} \quad J \quad$ Haematol. 2005;128(5):698-702. doi:10.1111/j.1365-2141.2004.05357.x

32. Ozcan Cetin EH, Cetin MS, Aras D, et al. Platelet to lymphocyte ratio as a prognostic marker of in-hospital and long-term major adverse cardiovascular events in st-segment elevation myocardial infarction. Angiology. 2016;67(4):336-345. doi:10.1177/0003319 715591751
33. Ferroni P, Basili S, Falco A, et al. Platelet activation in type 2 diabetes mellitus. J Thromb Haemost. 2004;2(8):1282-1291. doi:10.1111/j.1538-7836.2004.00836.x

34. Frangogiannis NG, Smith CW, Entman ML. The inflammatory response in myocardial infarction. Cardiovasc Res. 2002;53 (1):31-47. doi:10.1016/S0008-6363(01)00434-5

35. Dinc T, Polat Duzgun A, Kayilioglu SI, et al. Factors affecting mortality after major nontraumatic lower extremity amputation. Int J Low Extrem Wounds. 2016;15(3):227-231. doi:10.1177/153473 4616655924

36. Spark JI, Sarveswaran J, Blest N, et al. An elevated neutrophil-lymphocyte ratio independently predicts mortality in chronic critical limb ischemia. J Vasc Surg. 2010;52(3):632-636. doi:10.1016/j.jvs.2010.03.067

\section{Publish your work in this journal}

Diabetes, Metabolic Syndrome and Obesity: Targets and Therapy is an international, peer-reviewed open-access journal committed to the rapid publication of the latest laboratory and clinical findings in the fields of diabetes, metabolic syndrome and obesity research. Original research, review, case reports, hypothesis formation, expert opinion and commentaries are all considered for publication. The manuscript management system is completely online and includes a very quick and fair peer-review system, which is all easy to use. Visit http://www.dovepress.com/testimonials.php to read real quotes from published authors. 